\title{
Noise Propagation in Chemical Reaction Networks: Analysis of a Molecular Subtractor Module
}

\author{
Carlo Cosentino ${ }^{1}$, Costanzo Manes $^{2}$, Giovanni Palombo ${ }^{3}$, Pasquale Palumbo ${ }^{3}$
}

\begin{abstract}
The realization of embedded molecular control systems is a challenging aim in Synthetic Biology, where a major goal is to design synthetic biological circuits performing specific tasks. In this field, the novel emergent approach is to assemble the circuit in a modular fashion, possibly restraining reciprocal interactions from interconnected modules (zero-retroactivity). Within this framework, recent results have been proposed, dealing with the realization of an embedded subtractor module, with the idea of exploiting it in a more general chemical reaction network that resembles a classical control scheme. So far, this research has been carried out according to the deterministic approach. More sophisticated analysis requires the use of stochastic models, which play a paramount role in investigating noise propagation in chemical reaction networks, especially when the species copy number is low and the intrinsic stochasticity of the phenomena under investigation cannot be neglected. This note deals with a first analysis of the subtractor module, according to the stochastic approach. To this end, Chemical Master Equations are exploited to model one of the possible molecular circuits implementing the subtractor, and moment equations are written in order to evaluate how noise propagates with respect to different values of the inputs and different model parameter settings.
\end{abstract}

Index Terms-Chemical reaction networks, Stochastic approach, Moment equations

\section{INTRODUCTION}

A major research trend in Synthetic Biology aims at designing molecular reaction systems in a modular fashion [14], [17], [11]. Modularity can be considered a common denominator among the Control Systems and Synthetic Biology communities. Indeed, if modularity is a well-established requirement in control systems design (and in synthetic science in general, spanning from the many applicative fields of engineering and computer science), from the other hand in a seminal paper of Hartwell et al. in 1999 [18], it is clearly suggested to detect and analyse cellular functional modules to describe and unravel the complexity of biological organization. To this end, the design of embedded interconnected molecular reaction modules has taken great advantage from recent results aiming at reducing retroactivity, i.e. the undesired coupling effect occurring by the mutual interaction

\footnotetext{
1 Department of Experimental and Clinical Medicine, University of Magna Graecia, 88100 Catanzaro, Italy carlo.cosentino@unicz.it

${ }^{2}$ Department of Information Engineering, Computer Science, and Mathematics, University of L'Aquila, Via Vetoio, 67100 L'Aquila, L'Aquila, Italy costanzo.manes@univaq. it

3 Istituto di Analisi dei Sistemi ed Informatica "A. Ruberti", Consiglio Nazionale delle Ricerche (IASI-CNR), Via dei Taurini 19, 00185 Roma, Italy giovanni.palombodiasi.cnr.it, pasquale.palumbo@iasi.cnr.it
}

of interconnected modules, which prevents their nominal behavior occurring as stand-alone devices [13].

The development of modular embedded molecular systems has fostered the application of control design concepts to realize and improve the behavior of synthetic circuits (see, e.g. [12] and references therein). Within this framework, a novel interesting trend is to design Chemical Reaction Networks (CRN) to work as modular building blocks, performing a specific task to be exploited when interconnected into a larger embedded system. This approach enables the development of a general method for the design of closedloop feedback control schemes in synthetic biology (like, e.g. the ones recently presented in [5], [15], [27], [23], [6], [25], [28]) by means of the most suitable selection and proper interconnection of distinct building blocks (similarly to classical control scheme design), each provided by the chosen CRN.

Encouraging results towards these directions have been presented in [9], where some CRN have been suggested for the realization of a subtractor module, able to compare (and make the difference of) a pair of input signals. Together with retroactivity analysis performed in [2], the design of a subtractor appears to play a pivotal role for the implementation of any modular feedback control scheme. In both papers a pure deterministic approach is exploited to model the CRN, according to the mass-action law. On the other hand, noise is known to play a relevant role in biological processes [22]. Random fluctuations, provided by a wide set of concurring factors including, for instance, thermal noise or asynchronous occurrence of synthesis and degradation events, need to be considered when modeling most of the molecular processes involved in cellular regulation, as well as in gene expression, see e.g. [1], [7]. Moreover, thinking at the subtractor within a classical closed-loop control scheme, it may have a reference signal to be tracked as input therefore, asymptotically, the subtractor may work with small species copy numbers, a framework that usually requires a stochastic modeling approach [29].

This note investigates noise propagation in a CRN that implements a subtractor module, according to a stochastic modeling approach that relies on Chemical Master Equations (CME), describing the time-evolution of the probabilities of the involved chemical species [29]. Except for very special cases, finding the exact solution of a CME (even just looking for the steady-state solution) is an extremely high-dimensional problem, not allowing affordable solutions in reasonable computational times. As a matter of fact, one usually resorts to deal with only a limited number of 
features related to the CME solution. Among these, second order moments are usually exploited to quantify how noise propagates within the CRN under investigation, according to the coefficient of variation associated to the given species (see, e.g. [24], [4]). These computations do not usually provide analytical solutions, since moment equations are not in closed-form unless the propensities associated to the CME are linear [19]. This drawback is here overcome by means of a moment closure techniques that has been proven to be especially suited for reactions with small population sizes [26]. Approximate analytical results are, then, compared to numerical Monte Carlo simulations, run according to the Gillespie Stochastic Simulation Algorithm (SSA) [16]: as usual, these numerical simulations (though time-consuming) will be considered as the baseline to validate the approximation provided by moment closure.

\section{Subtractor MOdel Setting}

The chemical reaction scheme under investigation is a version of the subtractor module proposed in [9]. It is an open Chemical Reaction Network (CRN) with 3 species, namely $\mathrm{A}, \mathrm{B}$ and $\mathrm{C}$. In the following, the species copy numbers will be referred to with $\mathbf{a}, \mathbf{b}$ and $\mathbf{c}$, respectively. Moreover, we will denote with $\langle h(\mathbf{a})\rangle$ the first order moment of a generic function $h$ of a random process a, and with $\overline{\langle h(\mathbf{a})\rangle}=\lim _{t \mapsto+\infty}\langle h(\mathbf{a})\rangle$ its stationary value whenever it exists and is finite. In case of the identity function $h(\mathbf{a})=\mathbf{a}$, its steady-state first order moment will be denoted by $\overline{\langle\mathbf{a}\rangle}=\overline{\mathbf{a}}$ for short.

The CRN species evolve according to four reactions: A species production, regulated by the exogenous productions rate $u_{1}$ (reaction 1 ), $\mathrm{B}$ species production regulated by the exogenous productions rate $u_{2}$ (reaction 2), degradation of species $\mathrm{A}$ and $\mathrm{B}$ with kinetic constant $k_{3}$ (reaction 3), and $\mathrm{C}$ species production as coming from the A-into-C transformation with kinetic constant $k_{4}$ (reaction 4):

$$
\varnothing \stackrel{1}{\rightarrow} \mathrm{A} \stackrel{4}{\rightarrow} \mathrm{C} \quad \varnothing \stackrel{2}{\rightarrow} \mathrm{B} \quad \mathrm{A}+\mathrm{B} \stackrel{3}{\rightarrow} \varnothing
$$

Dealing with the CRN as an input/output plant, we set the two inputs in the exogenous fluxes $u_{1}, u_{2}$, while the chosen output is given by species $\mathrm{C}$ flux. Indeed, fluxes are known to be the interconnection signals of CRNs. According to the deterministic approach, in [9] it is shown that such a CRN satisfies the properties that ensure the output flux of $\mathrm{C}$ to converge asymptotically to the difference of the two input fluxes $u_{1}-u_{2}$, provided that the input fluxes are constant and $u_{1}>u_{2}$.

According to the stochastic approach, Table I describes the discrete resets associated to the reaction set (1), as well as the corresponding propensities $w_{i}, i=1,2,3,4$ that allow one to build the Chemical Master Equations associated to the CRN. The degradation process is treated similarly to a sequestration dynamics, with the propensity modeled by the product of the binding species copy number (see, e.g. [8], [10], [21]). CMEs consist of a set of Ordinary Differential Equations (ODE) describing the probability associated to any discrete combination of the species copy numbers [29]. CMEs are
TABLE I

CHEMICAL REACTIONS.

\begin{tabular}{|c|c|c|c|}
\hline Reaction ID & Event & Reset & Propensity function \\
\hline 1 & A production & $\mathbf{a} \mapsto \mathbf{a}+1$ & $w_{1}=u_{1}$ \\
\hline 2 & B production & $\mathbf{b} \mapsto \mathbf{b}+1$ & $w_{2}=u_{2}$ \\
\hline 3 & A, B clearance & $\mathbf{a} \mapsto \mathbf{a}-1$ & $w_{3}(\mathbf{a}, \mathbf{b})=k_{3} \mathbf{a b}$ \\
& & $\mathbf{b} \mapsto \mathbf{b}-1$ & \\
\hline 4 & C production & $\mathbf{c} \mapsto \mathbf{c}+1$ & $w_{4}(\mathbf{a})=k_{4} \mathbf{a}$ \\
& & $\mathbf{a} \mapsto \mathbf{a}-1$ & \\
\hline
\end{tabular}

known to properly deal with fluctuations and chemical fluxes, and they are usually invoked whenever a deeper information concerning noise investigation and propagation is required. The drawback of such a powerful modeling tool is the so called curse of dimensionality which, in many cases, although CMEs are linear equations, prevents from explicitly computing the solutions. Therefore, even when looking for the stationary distribution, one usually sets the problem in order to implement efficient algorithms [3], or resort to approximate solutions (like e.g. the Finite State Projection algorithm [20]), or to numerical Monte Carlo approaches, like the Gillespie Stochastic Simulation Algorithm (SSA) [16].

In this note we exploit the CME to achieve the first- and second-order moments equations. First-order moments provide the average values of the species copy numbers, while second-order moments allow to quantify species fluctuations, thus providing a measurement of noise propagation. In fact, we are interested in assessing the fluctuations of the model output provided by the flux of species $C$. Then, according to the stochastic approach, such an output $y$ is provided by the propensity of reaction 4 , namely $y=w_{4}(\mathbf{a})=k_{4}$ a. Similarly to [24], [4] we exploit the stationary Coefficient of Variation (CV) to measure the output noise, therefore

$$
C V_{y}^{2}=\frac{\overline{\left\langle w_{4}^{2}(\mathbf{a})\right\rangle}-\left(\overline{w_{4}(\mathbf{a})}\right)^{2}}{\left(\overline{w_{4}(\mathbf{a})}\right)^{2}}=\frac{\overline{\left\langle\mathbf{a}^{2}\right\rangle}-\overline{\mathbf{a}}^{2}}{\overline{\mathbf{a}}^{2}}
$$

Any order moment equations can be readily written because the model nonlinearities are of polynomial fashion [19]. Moment equations are linear but, unfortunately, they are not in closed form, in the sense that lower-order moment equations depend on higher order moments, unless all propensities are linear. As a matter of fact any order moment equations can be thought of as linearly evolving on an infinite-dimensional state space. This drawback is overcome by approximating higher order terms by suitably defined nonlinear functions of lower order moments [26]. Following this moment closure technique we provide the analytical solution of the approximate moment equation in the next Section.

\section{Moments EQuATions}

It is worth noting that the output of the system under investigation (i.e. the flux of $\mathrm{C}$ provided by the propensity of reaction 4) is univocally identified by the copy number of 
only species A and B, evolving independently of species $\mathrm{C}$. Therefore, in the following, from an input/output perspective, we will refer to the $C$ flux as the output of the subsystem of species $\mathrm{A}$ and $\mathrm{B}$.

By denoting with $\mathbf{x}=\left[\begin{array}{ll}\mathbf{a} & \mathbf{b}\end{array}\right]^{T}$ the aggregate vector of the A, B species copy numbers, the general formula to achieve the dynamics of any order moment of the type $\mathbf{a}^{i_{1}} \mathbf{b}^{i_{2}}$ from the model is below reported [19]

$$
\frac{d}{d t}\left\langle\psi_{i_{1}, i_{2}}(\mathbf{x})\right\rangle=\sum_{j=1}^{4}\left\langle\left(\psi_{i_{1}, i_{2}}\left(\mathbf{x}+\Delta_{j}\right)-\psi_{i_{1}, i_{2}}(\mathbf{x})\right) w_{j}(\mathbf{x})\right\rangle
$$

where

$$
\psi_{i_{1}, i_{2}}(\mathbf{x})=\mathbf{a}^{i_{1}} \mathbf{b}^{i_{2}}, \quad i_{1}, i_{2}=0,1, \ldots
$$

is a polynomial function implementing any order moment by properly varying the integers $i_{1}, i_{2}$, and $\Delta_{j}$ stands for the state update that happens whenever reaction $j$ occurs. For instance, the four reactions in Table $\mathrm{I}$ are described by:

$$
\Delta_{1}=\left[\begin{array}{l}
1 \\
0
\end{array}\right], \quad \Delta_{2}=\left[\begin{array}{l}
0 \\
1
\end{array}\right], \quad \Delta_{3}=\left[\begin{array}{l}
-1 \\
-1
\end{array}\right], \quad \Delta_{4}=\left[\begin{array}{c}
-1 \\
0
\end{array}\right] \text {. }
$$

Therefore, first-order moments are readily computed for $\psi_{1,0}(\mathbf{x})=\mathbf{a}$ and $\psi_{0,1}(\mathbf{x})=\mathbf{b}$ :

$$
\begin{aligned}
& \frac{d}{d t}\langle\mathbf{a}\rangle=u_{1}-k_{3}\langle\mathbf{a b}\rangle-k_{4}\langle\mathbf{a}\rangle \\
& \frac{d}{d t}\langle\mathbf{b}\rangle=u_{2}-k_{3}\langle\mathbf{a b}\rangle
\end{aligned}
$$

It is easy to check that at steady-state we get

$$
\overline{\langle\mathbf{a b}\rangle}=\frac{u_{2}}{k_{3}}, \quad \overline{\mathbf{a}}=\frac{u_{1}-u_{2}}{k_{4}}
$$

which imply that the output $y=k_{4} \mathbf{a}$ asymptotically converges in average to the displacement $u_{1}-u_{2}$. It is worth noting that such results are achieved without any approximation and it confirms in average the same results yielded by the deterministic approach.

Unfortunately, due to the nonlinearity of one of the propensities there is not a closed form for the other steadystate first-order moment, i.e. $\overline{\mathbf{b}}$, therefore we need to resort to moment closure techniques. It is also apparent that species $\mathrm{C}$ does not converge in average to a finite copy number, since the model does not account for any clearance reaction for $\mathrm{C}$.

Concerning the second-order moment dynamics we consider $\psi_{2,0}(\mathbf{x})=\mathbf{a}^{2}, \psi_{0,2}(\mathbf{x})=\mathbf{b}^{2}$ and $\psi_{1,1}(\mathbf{x})=\mathbf{a b}$ in (3) so that:

$$
\begin{aligned}
& \frac{d}{d t}\left\langle\mathbf{a}^{2}\right\rangle=-2 k_{4}\left\langle\mathbf{a}^{2}\right\rangle+k_{3}\langle\mathbf{a b}\rangle-2 k_{3}\left\langle\mathbf{a}^{2} \mathbf{b}\right\rangle \\
&+\left(2 u_{1}+k_{4}\right)\langle\mathbf{a}\rangle+u_{1} \\
& \frac{d}{d t}\left\langle\mathbf{b}^{2}\right\rangle=k_{3}\langle\mathbf{a b}\rangle+2 u_{2}\langle\mathbf{b}\rangle+u_{2}-2 k_{3}\left\langle\mathbf{a b}^{2}\right\rangle \\
& \frac{d}{d t}\langle\mathbf{a b}\rangle=\left(k_{3}-k_{4}\right)\langle\mathbf{a b}\rangle+u_{1}\langle\mathbf{b}\rangle+u_{2}\langle\mathbf{a}\rangle \\
&-k_{3}\left\langle\mathbf{a}^{2} \mathbf{b}\right\rangle-k_{3}\left\langle\mathbf{a b}^{2}\right\rangle
\end{aligned}
$$

Again, we obtain second-order moment equations that do not provide closed-form solutions because of the model nonlinearities. To solve them we need to exploit moment closure approximations [26]:

$$
\left\langle\mathbf{a}^{2} \mathbf{b}\right\rangle \cong \frac{\left\langle\mathbf{a}^{2}\right\rangle}{\langle\mathbf{b}\rangle}\left(\frac{\langle\mathbf{a b}\rangle}{\langle\mathbf{a}\rangle}\right)^{2}, \quad\left\langle\mathbf{a b}^{2}\right\rangle \cong \frac{\left\langle\mathbf{b}^{2}\right\rangle}{\langle\mathbf{a}\rangle}\left(\frac{\langle\mathbf{a b}\rangle}{\langle\mathbf{b}\rangle}\right)^{2}
$$

After substituting the nonlinear functions (12) in 97-(11) we obtain a closed nonlinear ODE, whose steady state solutions come out from the following algebraic system:

$$
\begin{aligned}
0= & -k_{4} \overline{\left\langle\mathbf{a}^{2}\right\rangle}+\frac{u_{1}\left(u_{1}-u_{2}\right)}{k_{4}}+u_{1}-\frac{\overline{\left\langle\mathbf{a}^{2}\right\rangle}}{\overline{\mathbf{b}}} \frac{u_{2}^{2} k_{4}^{2}}{k_{3}\left(u_{1}-u_{2}\right)^{2}} \\
0= & +\overline{\mathbf{b}}-k_{4} \frac{\overline{\left\langle\mathbf{b}^{2}\right\rangle}}{u_{1}-u_{2}} \frac{u_{2}}{k_{3} \overline{\mathbf{b}}^{2}} \\
0= & u_{2} \frac{k_{3}-k_{4}}{k_{3}}+u_{1} \overline{\mathbf{b}}+u_{2} \frac{u_{1}-u_{2}}{k_{4}} \\
& -\frac{\overline{\left\langle\mathbf{a}^{2}\right\rangle}}{\overline{\mathbf{b}}} \frac{u_{2}^{2} k_{4}^{2}}{k_{3}\left(u_{1}-u_{2}\right)^{2}}-k_{4} \frac{\overline{\left\langle\mathbf{b}^{2}\right\rangle}}{u_{1}-u_{2}} \frac{u_{2}^{2}}{k_{3} \overline{\mathbf{b}}^{2}}
\end{aligned}
$$

By properly manipulating these constraints, we obtain

$$
0=-u_{2} \frac{k_{4}}{k_{3}}+\left(u_{1}-u_{2}\right) \overline{\mathbf{b}}-u_{1}-\frac{\left(u_{1}-u_{2}\right)^{2}}{k_{4}}+k_{4} \overline{\left\langle\mathbf{a}^{2}\right\rangle}
$$

and so we can write $\overline{\left\langle\mathbf{a}^{2}\right\rangle}$ as a function of $\overline{\mathbf{b}}$ :

$$
\overline{\left\langle\mathbf{a}^{2}\right\rangle}=\frac{u_{1}}{k_{4}}+\frac{u_{2}}{k_{3}}+\left(\frac{u_{1}-u_{2}}{k_{4}}\right)\left(\frac{u_{1}-u_{2}}{k_{4}}-\overline{\mathbf{b}}\right)
$$

By substituting (17) in (13) leads to the quadratic equation in $\bar{b}$ with the following form

$$
\alpha \overline{\mathbf{b}}^{2}+\beta \overline{\mathbf{b}}+\gamma=0
$$

where

$$
\begin{aligned}
& \alpha=u_{1}-u_{2} \\
& \beta=-\frac{u_{2}^{2}}{k_{4}}+\frac{u_{1} u_{2}}{k_{4}}-\frac{k_{4}}{k_{3}} u_{2}+\frac{k_{4}}{k_{3}} \frac{u_{2}^{2}}{u_{1}-u_{2}} \\
& \gamma=-k_{3}\left(\frac{u_{1}}{k_{4}}+\frac{u_{2}}{k_{3}}+\left(\frac{u_{1}-u_{2}}{k_{4}}\right)^{2}\right)\left(\frac{u_{2} k_{4}}{k_{3}\left(u_{1}-u_{2}\right)}\right)^{2}
\end{aligned}
$$

It is readily verified that, since $\alpha>0$ and $\gamma<0$, there always exist two real solutions, one positive and one negative, since $\beta^{2}-4 \alpha \gamma>0$ and $\beta^{2}-4 \alpha \gamma>\beta^{2}$, that means there exists a unique meaningful (i.e positive real) solution for stationary $\overline{\mathrm{b}}$ :

$$
\overline{\mathbf{b}}=\frac{-\beta+\sqrt{\beta^{2}-4 \alpha \gamma}}{2 \alpha}
$$

From (13) and (14), according to (21), we get

$$
\begin{aligned}
\overline{\left\langle\mathbf{b}^{2}\right\rangle} & =(1+\overline{\mathbf{b}}) \frac{k_{3} \overline{\mathbf{b}}^{2}\left(u_{1}-u_{2}\right)}{k_{4} u_{2}} \\
\overline{\left\langle\mathbf{a}^{2}\right\rangle} & =\frac{k_{3} \overline{\mathbf{b}} u_{1}\left(u_{1}-u_{2}\right)^{2}\left(k_{4}+u_{1}-u_{2}\right)}{k_{4}^{2}\left(k_{3}\left(u_{1}-u_{2}\right)^{2} \overline{\mathbf{b}}+k_{4} u_{2}^{2}\right)}
\end{aligned}
$$

And finally, by properly exploiting (2) we have the following CV for the output:

$$
C V_{y}^{2}=\frac{k_{3} k_{4} \overline{\mathbf{a}} \overline{\mathbf{b}}+k_{3} \overline{\mathbf{b}}(1+\overline{\mathbf{a}}) u_{2}-u_{2}^{2}}{k_{3} k_{4} \overline{\mathbf{b}} \overline{\mathbf{a}}^{2}+u_{2}^{2}}
$$




\section{Discussion}

This Section aims at investigating how noise fluctuations impact the output of the subtractor according to different working modes and/or model parameter settings. As a preliminary remark, differently from what happens for the firstorder moments of the subtractor output (depending only on the fourth reaction propensity, equal at steady-state to the difference $u_{1}-u_{2}$ ), both $\overline{\mathbf{b}}$ and the second-order moment (and the output $C V_{y}$ as well) depend on both $u_{1}$ and $u_{2}$.

\section{A. Uniform variations of both input fluxes}

Assume to uniformly vary $u_{1}$ and $u_{2}$ so that the difference $\delta=u_{1}-u_{2}$ is kept fixed. This way one can investigate what happens to the subtractor output fluctuations when the input fluxes increase (or decrease) according to different output working modes (i.e. according to different output average values). To answer this question we first consider how $\overline{\mathbf{b}}$ varies. Then, let $u_{2}$ vary and fix $u_{1}=u_{2}+\delta$. According to (18)-21 it can be shown, after computations, that

$$
\lim _{u_{2} \mapsto 0^{+}} \overline{\mathbf{b}}=0, \quad \lim _{u_{2} \mapsto+\infty} \frac{\overline{\mathbf{b}}}{u_{2}}=\frac{k_{3}+k_{4}}{\delta k_{3}} .
$$

The first limit in 25] is trivially coherent with the hypothesis that, without the input $u_{2}$, there is no accumulation of B. On the other hand, the second limit tells us that $\overline{\mathbf{b}}$ definitely diverges to $+\infty$ with $u_{2}$ according to a linear fashion. The slope of the oblique asymptote tells us about the asymptotic sensitivity of $\mathbf{b}$ with respect to $u_{2}$ : the greater is the slope, the greater is the asymptotic variation of $\bar{b}$ with respect to variations of $u_{2}$.

As for the Coefficient of Variation of the output (24), the previous analysis carried out on $\overline{\mathbf{b}}$ provides the following results. By varying $u_{2}$ when $\delta$ is kept constant, it happens that:

- if $u_{2} \mapsto 0^{+}$, then $C V_{y}^{2}$ converges to the following finite value:

$$
\lim _{u_{2} \mapsto 0^{+}} C V_{y}^{2}=\frac{k_{4}}{\delta}
$$

Therefore, in case of low input fluxes (keeping fixed $\delta$ ) the output noise fluctuations become proportional to parameter $k_{4}$ (and insensitive to parameter $k_{3}$ ). That means, in case of low input fluxes, the output noise reduction could be achieved by properly reducing the strength of reaction 4 ;

- if $u_{2} \mapsto+\infty$, then $C V_{y}^{2}$ converges to the following finite value:

$$
\lim _{u_{2} \mapsto+\infty} C V_{y}^{2}=\frac{k_{3}+k_{4}}{\delta}+\frac{k_{3}}{k_{4}}
$$

Therefore, in case of high input fluxes (keeping fixed $\delta$ ) the output noise fluctuations monotonically increase with $k_{3}$, whilst a non-monotonic behavior is referred to $k_{4}$.

Fig. 1 draws (as a continuous line) $C V_{y}^{2}$-vs- $u_{2}$ for different values of $\delta$.

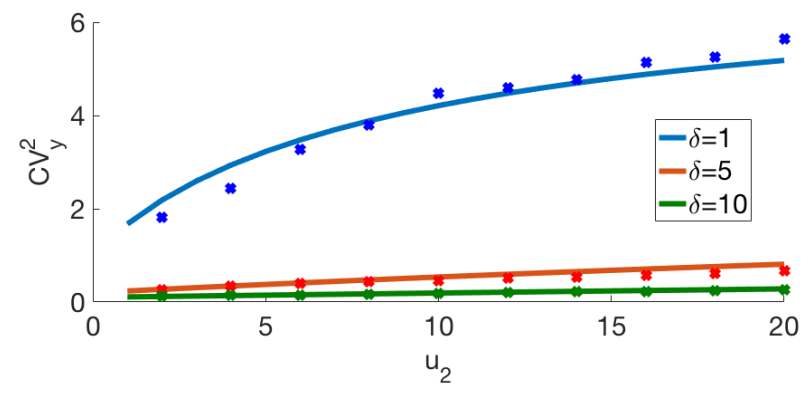

Fig. 1. $C V_{y}^{2}$ of the output of the subtractor module for different values of $u_{2}$, keeping fixed $\delta=u_{1}-u_{2}$, with parameters $k_{3}=3.1$ and $k_{4}=1.0$. Markers refer to numerical SSA.

\section{B. Input difference variations}

Assume to keep fixed $u_{2}$ and let $\delta$ (i.e. the output of the subtractor) vary. Then, we have the following limits for $\bar{b}$ :

$$
\lim _{\delta \mapsto 0^{+}} \overline{\mathbf{b}}=+\infty, \quad \lim _{\delta \mapsto+\infty} \overline{\mathbf{b}}=0 .
$$

The first limit in 28 substantially states that the steadystate average value $\overrightarrow{\mathbf{b}}$ tends to be large when $\delta$ is small (keeping constant $u_{2}$ flux); instead, the second limit states that $\overline{\mathbf{b}}$ decreases to zero when the difference $\delta$ goes to $+\infty$. This behavior is coherent with the kind of reactions, since by keeping fixed $u_{2}$ and reducing $\delta \mapsto 0^{+}$we force as well $\overline{\mathbf{a}} \mapsto 0$, according to 8 ; therefore, without A copy number, reaction 3 never occurs and thus there is no clearance for $\mathrm{B}$, that indefinitely accumulates according to a fixed input flux $u_{2}$. On the other hand, by increasing $\delta \mapsto+\infty$ we make as well $\overline{\mathbf{a}} \mapsto+\infty$, therefore the propensity of reaction 3 (the one related to the clearance of $\mathrm{B}$ ) increases as well to $+\infty$, thus forcing the asymptotic depletion of species $\mathrm{B}$.

By varying $\delta$ when $u_{2}$ is kept constant, it happens that if $\delta \mapsto 0^{+}$, then $C V_{y}^{2}$ diverges to $+\infty$ whilst if $\delta \mapsto+\infty$, then $C V_{y}^{2}$ converges to zero

$$
\lim _{\delta \mapsto 0^{+}} C V_{y}^{2}=+\infty \quad \lim _{\delta \mapsto+\infty} C V_{y}^{2}=0
$$

These results are reasonably coherent with the idea that a greater output is subject to a smaller noise and vice versa, regardless of the other model parameters. Fig. 2 draws (as a continuous line) $C V_{y}^{2}$-vs- $\delta$ for different values of $u_{2}$.

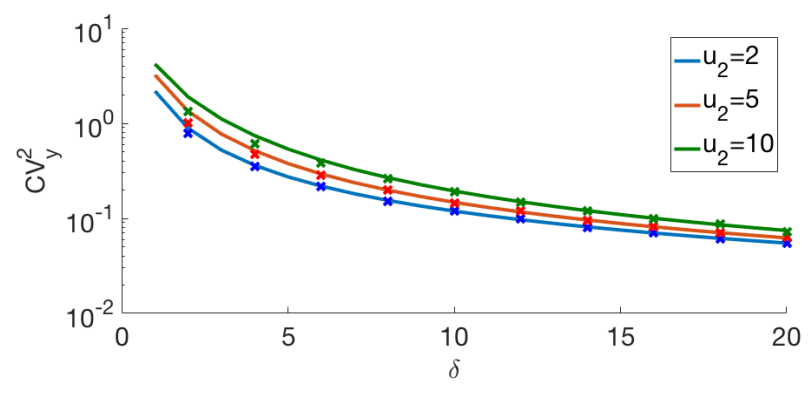

Fig. 2. $C V_{y}^{2}$ of the output of the subtractor module for different values of $\delta=u_{1}-u_{2}$, keeping fixed flux $u_{2}$, with parameters $k_{3}=3.1$ and $k_{4}=1.0$. Markers refer to numerical SSA. The $y$-axis is in logarithmic scale. 


\section{Strength of reaction 3 ( $A$ and $B$ clearance)}

By keeping fixed $u_{1}, u_{2}$ and $k_{4}$, and letting $k_{3}$ vary, the following limits occur:

$$
\lim _{k_{3} \mapsto 0^{+}} \overline{\mathbf{b}}=+\infty, \quad \lim _{k_{3} \mapsto+\infty} \overline{\mathbf{b}}=0,
$$

meaning that the steady-state average value $\overline{\mathbf{b}}$ tends to be large when the strength of reaction 3 reduces; on the other hand, when reaction 3 is dominant, then B is strongly forced to be cleared, thus providing a trivial stationary value.

Regards to the output noise, these results have the following implications. By decreasing the strength of chemicals degradation, the output noise decreases to the following lower bound:

$$
\lim _{k_{3} \mapsto 0^{+}} C V_{y}^{2}=\frac{k_{4}}{\delta}
$$

That means, low values of $k_{3}$ provide the same behavior (with respect to noise propagation) as for low values of $u_{2}$, with the coefficient of variation proportional to parameter $k_{4}$. Instead, by increasing the strength of chemicals degradation, the output noise increases up to the following upper bound:

$$
\lim _{k_{3} \mapsto+\infty} C V_{y}^{2}=\frac{k_{4}}{\delta}\left(1+\frac{u_{2}}{\delta}\right)
$$

It is worth noting that for high values of the output the two bounds become closer and closer, thus making the subtractor substantially insensitive to the strength of $k_{3}$.

Fig. 3 draws (as a continuous line) $C V_{y}^{2}$-vs- $k_{3}$ for different choices of $u_{1}$ and $u_{2}$.

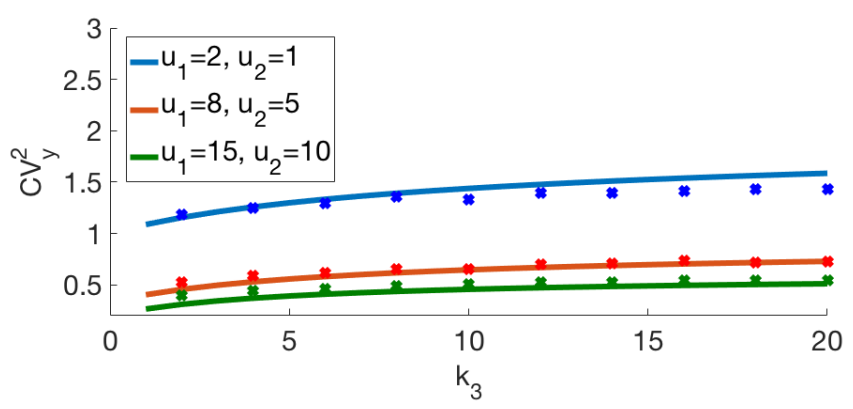

Fig. 3. $C V_{y}^{2}$ of the output of the subtractor module for different values of parameter $k_{3}$, keeping fixed fluxes $u_{1}, u_{2}$, and parameter $k_{4}=1.7$. Markers refer to numerical SSA.

\section{Strength of reaction 4 (C production)}

By keeping fixed $u_{1}$ and $u_{2}$ and $k_{3}$, and letting $k_{4}$ vary, the following limits occur:

$$
\lim _{k_{4} \mapsto 0^{+}} \overline{\mathbf{b}}=0, \quad \lim _{k_{4} \mapsto+\infty} \overline{\mathbf{b}}=+\infty,
$$

Indeed, by reducing reaction 4 strength $\left(k_{4}\right)$, keeping fixed the inputs, we have that the stationary value $\overline{\mathbf{a}}$ tends to be large, see (8), thus providing a large propensity for the clearance reaction associated to $\mathrm{B}$. On the other hand, the steadystate average value $\overline{\mathbf{b}}$ tends to be large when reaction 4 is dominant, then $\mathrm{A}$ is strongly forced to transform into $\mathrm{C}$, thus reducing its stationary copy number to a negligible value, therefore providing a negligible value for the propensity of the clearance reaction associated to $\mathrm{B}$ (thus $\overline{\mathrm{b}}$ diverges to $+\infty)$.

Concerning the output noise, by keeping fixed $u_{1}, u_{2}$ and $k_{3}$, and letting $k_{4}$ vary, if $k_{4} \mapsto 0^{+}$, then $C V_{y}^{2}$ converges to zero, whilst if $k_{4} \mapsto+\infty$, then $C V_{y}^{2}$ diverges to $+\infty$ :

$$
\lim _{k_{4} \mapsto 0^{+}} C V_{y}^{2}=0 \quad \lim _{k_{4} \mapsto+\infty} C V_{y}^{2}=+\infty
$$

therefore, in case of high values of $k_{4}$ the output noise fluctuations become larger and larger, thus making less reliable the output of the subtractor. The opposite happens for low values of $k_{4}$, i.e. a larger output corresponding to a smaller noise. Fig. 4 draws (as a continuous line) $C V_{y}^{2}$-vs- $k_{4}$ for different choices of $u_{1}$ and $u_{2}$.

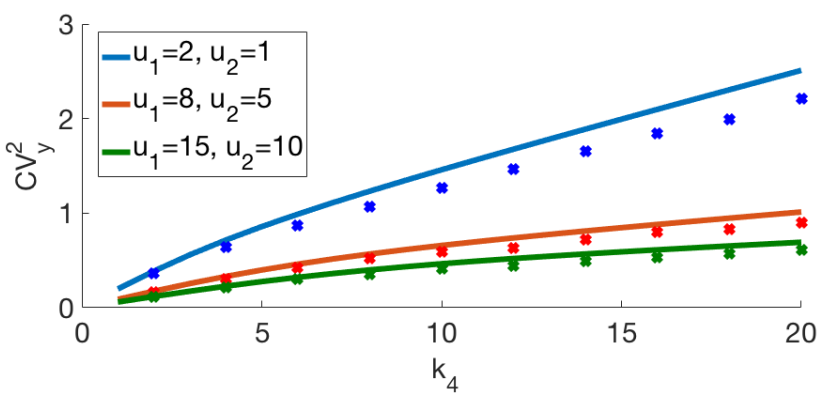

Fig. 4. $C V_{y}^{2}$ of the output of the subtractor module for different values of parameter $k_{4}$, keeping fixed fluxes $u_{1}, u_{2}$, and parameter $k_{3}=1.1$. Markers refer to numerical SSA.

In summary, we may say that:

- the stationary average output value is provided by 8 without any approximation, and it matches the results derived through the deterministic approach, constraining it to the difference of the two input fluxes;

- regards to the output noise, analytical computations, leveraging the moment closure approximation, show that noise reduction may be obtained in different ways: (i) the greater the subtractor output, the smaller the output noise, whatever the other model parameter configurations; (ii) small values of $k_{4}$ help to reduce the output noise, though in some cases $C V_{y}^{2}$ exhibits a nonmonotonic dependence on $k_{4}$, e.g. in presence of high input fluxes, see (27).

To test the validity of the moment closure approximations (12) exploited to achieve the analytical expression of firstand second-order moments, we run a set of numerical Gillespie simulations (SSA). All figures report markers that refer to a numerical SSA, showing the good performances of the moment closure approximation, thus validating the proposed theoretical results.

\section{CONCLUSiOnS}

A molecular subtractor module has been investigated in order to analyse noise propagation. To this end, a stochastic approach has been followed and Chemical Master Equations have been considered to model the Chemical Reaction Network that realizes the subtractor, and to evaluate the moment 
equations of the system. Moment closure approximation techniques have been exploited in order to achieve analytical results in terms of the Coefficient of Variation of the output of the system, providing a measurement of noise fluctuations around the steady-state value. Explicit solutions allow to understand the role of the inputs and of the model parameters in noise amplification or attenuation. Numerical simulations based on the Gillespie SSA confirm the validity of the approximation of the analytical results.

\section{ACKNOWLEDGEMENTS}

PP and GP are supported by the MIUR grant SysBioNet Italian Roadmap for ESFRI Research Infrastructures, SYSBIO Centre of Systems Biology, Milan and Rome, Italy.

\section{REFERENCES}

[1] R. Bahar, C.H. Hartmann, K.A. Rodriguez, A.D. Denny, R.A. Busuttil, M.E. Dollé, R.B. Calder, G.B. Chisolm, B.H. Pollock, C.A. Klein, and $\mathrm{J}$. Vijg, Increased cell-to-cell variation in gene expression in ageing mouse heart, Nature, 441, pp. 1011-1014, 2006.

[2] M. Bilotta, C. Cosentino, A. Merola, D.G. Bates, and F. Amato, Zeroretroactivity subtraction module for embedded feedback control of chemical reaction networks, IFAC-PapersOnLine, 49(26), pp. 128-133, 2016

[3] A. Borri, F. Carravetta, G. Mavelli, and P. Palumbo, Block-tridiagonal state-space realization of Chemical Master Equations: A tool to compute explicit solutions, Journal of Computational and Applied Mathematics, 296, pp. 410-426, 2016.

[4] A. Borri, P. Palumbo, and A. Singh, Impact of negative feedback in metabolic noise propagation, IET Syst. Biol., pp. 1-8, 2016.

[5] C. Briat, A. Gupta, and M. Khammash, Antithetic integral feedback ensures robust perfect adaptation in noisy biomolecular networks, Cell systems, 2(1), pp. 15-26, 2016.

[6] C. Briat, A. Gupta, and K. Khammash, Antithetic proportional-integral feedback for reduced variance and improved control performance of stochastic reaction networks, J.R. Soc. Interface, 15: 20180079, 2018.

[7] F.J. Bruggeman, N. Blüthgen, and H.V. Westerhoff, Noise management by molecular networks, PLoS Computational Biology, 5(9), e1000506, 2009.

[8] N.E. Buchler, and M. Louis, Molecular titration and ultrasensitivity in regulatory networks, J. Mol. Biol., 384, pp. 1106-1119, 2008.

[9] C. Cosentino, R. Ambrosino, M. Ariola, M. Bilotta, A. Pironti, and F. Amato, On the realization of an embedded subtractor module for the control of chemical reaction networks, IEEE Trans. Automatic Control, 61(11), pp. 3638-3643, 2016.

[10] M. Del Giudice, C. Bosia, S. Grigolon, and S. Bo, Stochastic sequestration dynamics: a minimal model with extrinsic noise for bimodal distributions and competitors correlation, Scientific Reports, 8:10387, 2018.

[11] D. Del Vecchio, Modularity, context-dependence, and insulation in engineered biological circuits, Trends in biotechnology, 33(2), pp. 111119,2015

[12] D. Del Vecchio, A.J. Dy, and Y. Qian, Control theory meets synthetic biology, Journal of The Royal Society Interface, 13(120), 20160380, 2016.

[13] D. Del Vecchio, A.J. Ninfa, and E.D. Sontag, Modular cell biology: retroactivity and insulation, Molecular Systems Biology, 4:161, 2008.

[14] D. Del Vecchio, E.D. Sontag, Engineering principles in bio-molecular systems: from retroactivity to modularity, European Journal of Control, 3-4, pp. 389-397, 2009.

[15] M.J. Dunlop, J.D. Keasling, and A. Mukhopadhyay, A model for improving microbial biofuel production using a synthetic feedback loop, Syst. Synth. Biol., 4, pp. 95-104, 2010.

[16] D.T. Gillespie, Exact Stochastic Simulation of Coupled Chemical Reactions, The Journal of Physical Chemistry, 81(25), pp. 2340-2361, 1977.

[17] A. Gyorgy, and D. Del Vecchio, Modular composition of gene transcription networks, PLoS Computational Biology, 10(3), e1003486, 2014.
[18] L.H. Hartwell, J.J. Hopfield, S. Leibler, and A.W. Murray, From molecular to modular cell biology, Nature, 402(6761 Supp), pp. 47-52, 1999.

[19] J.P. Hespanha, and A. Singh, Stochastic models for chemically reacting systems using polynomial stochastic hybrid systems, International Journal of robust and nonlinear control, 15(15), pp. 669-689, 2005.

[20] B. Munsky, and M. Khammash, The finite state projection approach for the analysis of stochastic noise in gene networks, IEEE Trans. Automat. Control, Special Issue on Systems Biology, pp. 201-214, 2008.

[21] Y.T. Lin, and N.E. Buchler, Efficient analysis of stochastic gene dynamics in the non-adiabatic regime using piecewise deterministic Markov processes, J.R. Soc. Interface, 15: 20170804, 2018.

[22] J.T. Mettetal, and E. Van Oudenaarden, Necessary Noise, Science, 317(5837), pp. 463-464, 2007.

[23] N. Olsman, A.-A. Baetica, F. Xiao, Y.P. Leong, R.M. Murray, and J.C. Doyle. Hard limits and performance tradeoffs in a class of sequestration feedback systems, bioRxiv, 2017.

[24] D.A. Oyarzun, J.-B. Lugagne, and G.-B.V. Stan, Noise propagation in synthetic gene circuits for metabolic control, ACS Synthetic Biology, 2014.

[25] C.C. Samaniego, and E. Franco. Ultrasensitive molecular controllers for quasi-integral feedback, bioRxiv, 2018.

[26] A. Singh, and J.P. Hespanha, Approximate moment dynamics for chemically reacting systems, IEEE Trans. Automatic Control, 56(2), pp. 414-418, 2011.

[27] J.A. Stapleton, K. Endo, Y. Fujita, K. Hayashi, M. Takinoue, H. Saito, and $\mathrm{T}$. Inoue, Feedback control of protein expression in mammalian cells by tunable synthetic translational inhibition, ACS Synth. Biol., 1, pp. 83-88, 2012.

[28] H. Steel, A.W.K. Harris, E.J. Hancock, C.L. Kelly, and A. Papachristodoulou, Frequency domain analysis of small non-coding RNAa shows summing junction-like behaviour, Proc. IEEE 56th Conf. Dec. Control, Melbourne, pp. 5328-5333, 2017.

[29] N.G. Van Kampen, Stochastic processes in physics and chemistry, 3rd ed. Amsterdam, The Netherlands: North Holland, 1992. 